\title{
Admissible cyclic representations and an algebraic approach to quantum phase
}

\author{
T Hakioglu \\ Physics Department Bilkent University, Ankara 06533, Turkey
}

Received 10 July 1997, in final form 9 October 1997

\begin{abstract}
Nonadmissible, weakly admissible and admissible cyclic representations and other algebraic properties of the generalized homographic oscillator (GHO) are studied in detail. For certain ranges of the deformation parameter, it is shown that this new deformed oscillator is a prototype cyclic oscillator endowed with a non-negative (admissible) spectrum. By changing the deformation parameter, the cyclic spectrum can be tuned to have an arbitrarily large period. It is shown that the standard harmonic oscillator is recovered at the nonadmissible infinite-period limit of the GHO. With these properties, the GHO provides a concrete example of an oscillator rich in a variety of cyclic representations. It is well known that such representations are of relevance to the proper algebraic formulation of the quantum-phase operator. Using a general scheme, it is shown that admissible cyclic algebras permit a well-defined Hermitian phase operator of which properties are studied in detail at finite periods as well as at the infinite-period limit. Fujikawa's index approach is applied to admissible cyclic representations and in particular to the phase operator in such algebras. Using the specific example of GHO it is confirmed that the infiniteperiod limit is distinctively singular. The connection with the Pegg-Barnett phase formalism is established in this singular limit as the period of the cyclic representations tends to infinity. The singular behaviour at this limit identifies the algebraic problems, in a concrete example, emerging in the formulation of a standard quantum harmonic-oscillator phase operator.
\end{abstract}

\section{Introduction}

Admissible cyclic representations are crucial for the rigorous formulation of the quantumphase operator. The classification of certain algebras which permit periodic action of the operators have been made by DeConcini and Kac [1]. Specific examples of $U_{q}(G)$ when $G$ defines a simple complex Lie algebra have been examined by Dobrev [2] and more recently by Abdesselam et al [3]. Periodic representations of generally deformed oscillators (in which Weyl-Heisenberg, Fibonacci and Biedenharn-Macfarlane oscillators are special cases) and their connection with the Hermitian quantum-phase problem has been recently noted by Fu and Sasaki [4]. In all known algebras which permit periodic representations with a period ( $M$ +1 ), the complex deformation parameter q becomes a root of unity. Two distinct cases are identified when $\mathrm{q}^{\mathrm{M}+1}=1$. In the first case the spectrum includes a zero and the spectral raising

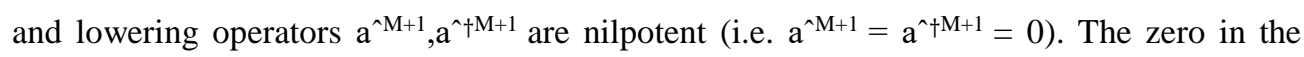
periodic spectrum is the eigenvalue of the vacuum state such that $\mathrm{a}^{\wedge}\left|0 \mathrm{i}={ }^{\wedge} \mathrm{a}^{\dagger}\right| \mathrm{Mi}=0$ and the periodic representations of these oscillators are similar to the regular representation of the 
harmonic oscillator using the Fock state. These are weakly admissible cyclic representations (ACR) within the context of this work. In the second case, in which the periodic spectrum does not involve a zero, the generally deformed oscillator can have admissibly cyclic representations such that $a^{\wedge+1}=a^{\wedge+M+1}$

0305-4470/98/020707+15\$19.50 C 1998 IOP Publishing Ltd

and the operators $\mathrm{a}^{\wedge \mathrm{M}+1}$ and $\mathrm{a}^{\wedge+\mathrm{M}+1}$ become the central elements of the generally deformed oscillator algebra.

In terms of a periodic representation, a generally deformed oscillator can be described by the action of the raising, $\mathrm{a}^{\wedge \dagger}$, and lowering, $\mathrm{a}^{\wedge}$, operators on the $(\mathrm{M}+1)$-dimensional Fock space $F_{M}$ as, $a^{\wedge}|n i=f(n) 1 / 2| n-1$ i n $6=0$

$$
\mathrm{a}^{\wedge}+\operatorname{ni}=\mathrm{f}(\mathrm{n}+1) 1 / 2 \mid \mathrm{n}+1 \mathrm{i} \quad \text { n } 6=\mathrm{M} \quad(1) \mathrm{a}^{\wedge}\left|0 \mathrm{i}=\mathrm{f}(0)_{1 / 2} \beta\right| \mathrm{Mi} \quad|\beta|=1 \mathrm{a}^{\wedge} \mid \mathrm{Mi}
$$

$=\mathrm{f}(\mathrm{M}+1)^{1 / 2} \beta^{*} \mid 0$ i Fsuch thateigenvalue of the generalized number operator $(\mathrm{N})^{\wedge} \mid \mathrm{ni}$

$=\mathrm{a}^{\wedge} \mathrm{f}(\mathrm{n}) \mathrm{F}(\mathrm{N})^{\wedge} \mid \mathrm{ni}=$, where $\mathrm{F}\left(\mathrm{N}^{\wedge} \mid \mathrm{n}+\mathrm{i}(\mathrm{n} 1)=\mathrm{a}^{\wedge}\right.$ 0and, $\left.1, \ldots, \mathrm{M}\right) \mathrm{a}^{\wedge}+\mathrm{F}\left(\mathrm{aN}^{\wedge}+\mathrm{a}^{\wedge}\right.$ are vectors in $\left.+=1 \mathrm{~F}\right)(=\mathrm{N})^{\wedge}$

Fand $(\mathrm{N})^{\wedge} \mathrm{Fa}^{\wedge} \mathrm{Maa}^{\wedge}+\mathrm{+}$. The. $=$ FHere, $\left(\left(\mathrm{NM}^{\wedge}++\mathrm{f}(\mathrm{n}) 11\right)\right)$ such thatperiodicis the

representations are implied by the cyclic property $\mathrm{F}(\mathrm{N})^{\wedge}=\mathrm{F}\left(\mathrm{N}^{\wedge}+\mathrm{M}+1\right)$ and

$$
\begin{aligned}
& \mathrm{a}^{\wedge}+\mathrm{M}+1 \mathrm{~F}(\mathrm{~N})^{\wedge}=\mathrm{F}\left(\mathrm{N}^{\wedge}+\mathrm{M}+1\right) \mathrm{a}^{\wedge}+\mathrm{M}+1=\mathrm{F}(\mathrm{N})^{\wedge} \mathrm{a}^{\wedge}+\mathrm{M}+1 \\
& \mathrm{~F}(\mathrm{~N})^{\wedge} \mathrm{a}^{\wedge \mathrm{M}+1}=\mathrm{a}^{\wedge \mathrm{M}+1} \mathrm{~F}\left(\mathrm{~N}^{\wedge}+\mathrm{M}+1\right)=\mathrm{a}^{\wedge \mathrm{M}+1} \mathrm{~F}(\mathrm{~N}) .^{\wedge}
\end{aligned}
$$

One also has the following relations

$a^{\wedge+M+1} a^{\wedge}\left|n i=f(n)^{1 / 2}\{f(n) f(n+1) \ldots f(n+M)\}^{1 / 2}\right| n+M i(3) a^{\wedge} a^{\wedge+M+1} \mid n i=f(n+M+1)^{1 / 2}\{f(n+$

1) $f(n+2) \ldots f(n+M+1)\}^{1 / 2} \mid n+M i$.

Comparing equations (3) we have [[a^ 1

elements of the corresponding $\left.a^{\wedge}+\right]=0$.

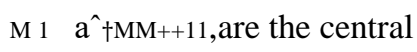
This result and equations (2) imply

algebra bywith a (non-negative) positive cyclic spectrum as $0_{+}$ ,F(N)^a,]a=†,F0.(N)^Thus,. We define (weakly) admissible cyclic representations (W)ACRa^ $+, \mathrm{a}^{\hat{\imath}}$

$6 \mathrm{f}(\mathrm{n})$. For those with positive as 
well as negative parts in the cyclic spectrum we use the nomenclature nonadmissible cyclic representations (NCR). Fu and Sasaki [4] have discussed the conditions for ACR of the generally deformed oscillators from the perspective of Hermitian quantum-phase operator. The generalized two-parameter Fibonacci oscillator

$$
[[\hat{N}]]=\frac{r_{1}^{\hat{N}}-r_{2}^{\hat{N}}}{r_{1}-r_{2}}
$$

does not permit ACR when $r_{1}$ and $r_{2}$ are roots of unity. The Biedenharn-Macfarlane oscillator and the naive q-deformed oscillator correspond to the specific examples of equation (4) when $r_{1}=r_{2}^{-1}$ and $r_{1} 6=r_{2}, r_{2}=1$ respectively. Hence the latter two oscillators also do not have ACR which is, in particular, crucial for the proper examination of the quantum phase operator.

In section 2, we will first examine the properties of all periodic representations of the generalized homographic oscillator (GHO) with particular emphasis on the admissible and weakly admissible ones. Section 3 is devoted to the formulation of an algebraic approach to the quantum-phase problem for a generally deformed oscillator with finite-dimensional (W)ACR. We then examine Fujikawa's index theorem which puts a stringent condition for the existence of an acceptable phase operator. It is shown that any WACR and ACR satisfy this index condition. The singularity at the limit $\mathrm{M}=\infty$ is explicitly shown by using the WACR and ACR of the generalized homographic oscillator. The connections are established with the harmonic-oscillator phase at this singular limit.

\section{The generalized homographic oscillator}

Recently, we have discussed a new species of the generalized deformed oscillator family, which, arises in the stereographic deformations of the invariant manifolds of the SU(2) and $\mathrm{SU}(1,1)$ algebras [5]. The algebra of this deformed oscillator is given by a generalized commutation relation in the form of a homographic function of the generalized number operator, hence, the name generalized homographic oscillator (GHO). The properties of the GHO in connection with the stereographic deformation of $\mathrm{SU}(2)$ and $\mathrm{SU}(1,1)$ have been examined in detail in [5]. The importance of this deformed oscillator lies in the fact that it naturally conforms to all the generic features of the periodic algebras enlisted in equations (1)-(3) in certain ranges of the deformation parameter which makes a useful algebraic tool to study the properties of quantum phase. The GHO can be parametrized by,

$$
\left(\begin{array}{l}
\alpha_{n+1} \\
\beta_{n+1}
\end{array}\right)=\mathcal{A}\left(\begin{array}{c}
\alpha_{n} \\
\beta_{n}
\end{array}\right) \quad \text { with } \mathcal{A}=\left(\begin{array}{ll}
p & 1 \\
k & 1
\end{array}\right)
$$

where $\mathrm{p}, \mathrm{k} \in \mathrm{R}$. Associating $\mathrm{f}(\mathrm{n})=\alpha_{\mathrm{n}} / \beta_{\mathrm{n}}$ with $\mathrm{f}(\mathrm{n}) \Leftrightarrow^{\wedge} \mathrm{a}^{\dagger} \mathrm{a}^{\wedge}$ and $\mathrm{f}(\mathrm{n}+1) \Leftrightarrow^{\wedge} \mathrm{a}^{\wedge} \uparrow$ the generalized commutation relation of the GHO algebra has been obtained in [5] as,

$$
\hat{a} \hat{a}^{\dagger}=\frac{p \hat{a}^{\dagger} \hat{a}+1}{k \hat{a}^{\dagger} \hat{a}+1} .
$$

We will consider the case $\operatorname{det} \mathrm{A}=1$ for simplicity. Of particular importance for the algebraic formulation of quantum-phase problems is the condition for a periodic spectrum. The eigenvalues of the matrix $A$ are,

$$
\lambda_{1,2}=\frac{1}{2}\left[\operatorname{tr}(\mathcal{A}) \pm \sqrt{[\operatorname{tr}(\mathcal{A})]^{2}-4 \operatorname{det}(\mathcal{A})}\right]=\left|\lambda_{1,2}\right| \mathrm{e}^{\mathrm{i} \varphi}
$$


where $\operatorname{tr}(\mathrm{A})=\mathrm{p}+1$ and $\operatorname{det}(\mathrm{A})=\mathrm{p}(\mathrm{M}-k$. The +1$)$-dimensional periodic spectrum is

obtained when the square root is 
From equation (11) it is clear that, the spectrum of NCR for the GHO are not evenly distributed in the entire range of allowed $\mathrm{k}$ values. They are the only ones present for $-26 \mathrm{k}$ $<0$ whereas they mix with the (W)ACR in the second range $-4<\mathrm{k}<-2$ (see below). The most important limiting case of NCR is $k=0^{-}$. As $k \rightarrow 0^{-}, r=1+\mathrm{i} \epsilon$ with $\epsilon=\sqrt{-k}$ describing a small parameter. It can be seen in equations (9) and (10) that, in this limit, both the Fibonacci oscillator and the GHO contract onto the standard harmonic oscillator (i.e. [[n]] $\rightarrow \mathrm{n}, \mathrm{f}(\mathrm{n}) \rightarrow \mathrm{n}$ ) with the spectral period approaching infinity. Namely, the harmonic oscillator is the infinite-period limit of the NCR corresponding to $\mathrm{k} \rightarrow 0^{-}$. As a severe consequence of that, an algebraic polar decomposition of the generators is not admitted and the search for unitarity of the phase operator becomes a hopeless task unless the algebraic properties are completely abandoned.

\subsection{Weakly admissible cyclic representations}

Although the cyclic representations of the underlying Fibonacci oscillator are always nonadmissible within the entire range $-4<\mathrm{k}<0$, weakly admissible ones are permitted for the GHO exclusively in the range $-4<\mathrm{k}<-2$, where, they coexist with NCR. For WACR, the spectrum is allowed to have a vacuum state (i.e. $\mathrm{f}(0)=0)$ but all other spectral eigenvalues are positively defined. In this range, WACR are associated with specific values of $\mathrm{k}$ which yield $[[n]] \propto(-1)^{n}$ so that $f(n)$ in equation (9) is always non-negative for $06 \mathrm{n}$. Since the vacuum state is present, $\mathrm{a}^{\wedge \mathrm{M}+1} ;{ }^{\wedge} \mathrm{a}^{\dagger \mathrm{M}+1}$ are nilpotent. The number of such

WACR for a particular M depends on the allowed values of $\mathrm{h}$. Since $-4<\mathrm{k}<-2$ implies $\frac{1}{2}<h /(M+1)<\frac{3}{2}$, the number of such representations for a particular $\mathrm{M}$ is $2(\mathrm{M}+1)$. As k $\rightarrow-4, \mathrm{M} \rightarrow \infty$ and WACR become increasingly dense. The spectral eigenvalues for a few $\mathrm{k}$ values are shown in figure 2 in the same phasor sheme of figure 1.

\subsection{Admissible cyclic representations}

For a general reference state $\mathrm{f}(0)$ the solution of equation (6) yields for the spectrum,

$$
f(n)=\frac{[1-f(0)][[n]]+f(0)[[n+1]]}{[1+k f(0)][[n]]-[[n-1]]}
$$


$\mathrm{h} / \mathrm{M}=1 / 304$

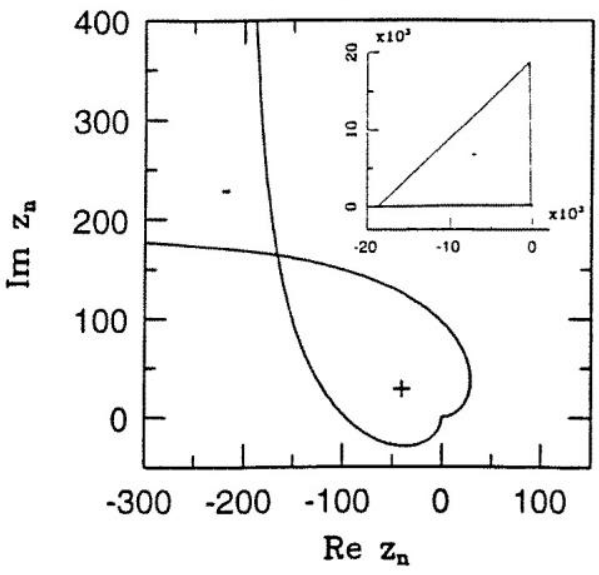

$\mathrm{h} / \mathrm{M}=2 / 304$

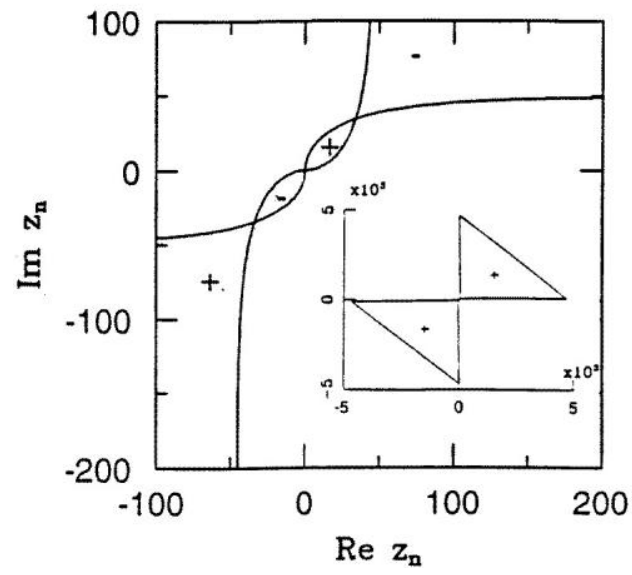

$h / M=3 / 304$

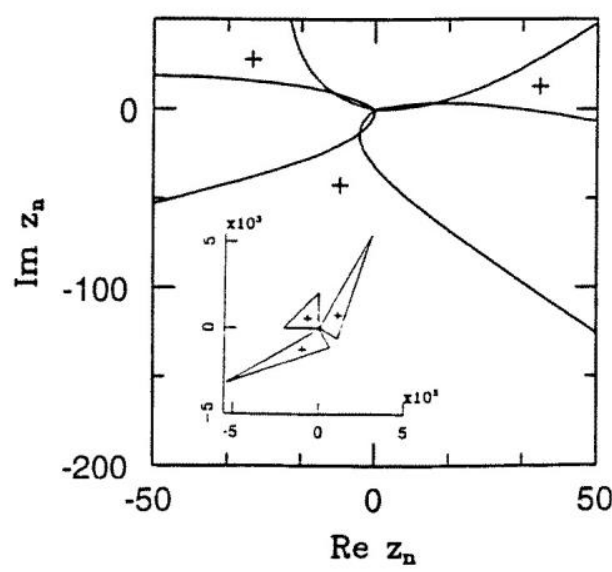

$h / M=4 / 304$

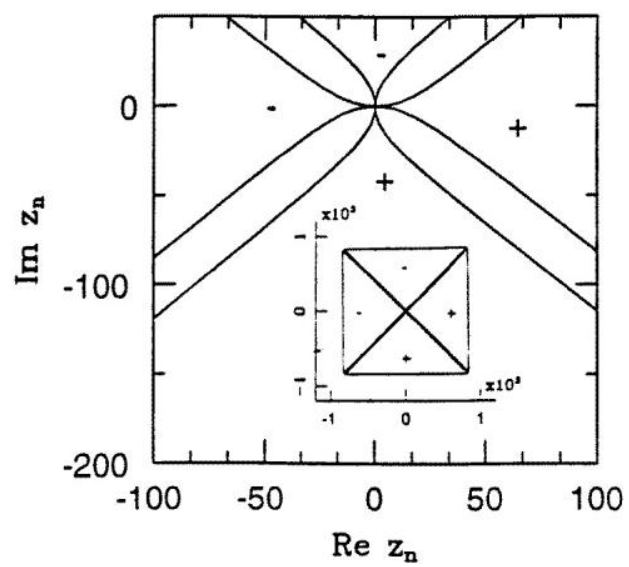

$z_{n}=f(n)^{1 / 2} \mathrm{e}^{\mathrm{i} n \varphi}$

crossing of $f(n)$

Figure 1. The closed trajectories of the spectral eigenvalues $f(n)$ in the phasor scheme of NCR. The sign of $f(n)$ are indicated within individual lobes. The zero are represented by the nodes. The main figures are exploded in the vicinity

of the origin in order to show how the sign change occurs for different winding numbers $\mathrm{h}=1,2,3,4$ and $\mathrm{M}=304$. Since, NCR are dense in the $\mathrm{k} \rightarrow 0$ - limit, we have chosen $\mathrm{h}, \mathrm{M}$ such that $h /(M+1) \ll 1$. The inlets are included to indicate the symmetry of the full trajectories.

where $[[n]]$ is still given by the Fibonacci oscillator spectrum in equation $(9)$. For $f(0)=0$ one recovers equation (9). The GHO described by the spectrum in equation (12) admits a large family of $\mathrm{ACR}$ in the range $-4<\mathrm{k}<-2$, with infinite variety of periods, where, the admissibility condition $0<f(n)$ is always satisfied in certain bands in the $f(0)-k$ space. For typical $M$ and $h$ values some of these bands are shown in figure 3 . For $f(0) 61$ there are forbidden zones where no admissible solution is found. Whereas, all solutions corresponding to $16 \mathrm{f}(0)$ are admissible for the same k values. In figure 4 the distribution of the spectral 
eigenvalues are plotted for certain $\mathrm{f}(0) 61$ and $16 \mathrm{f}(0)$ respectively as $\mathrm{M}$ and $\mathrm{h}$ are chosen properly to complement the picture in figure 3.
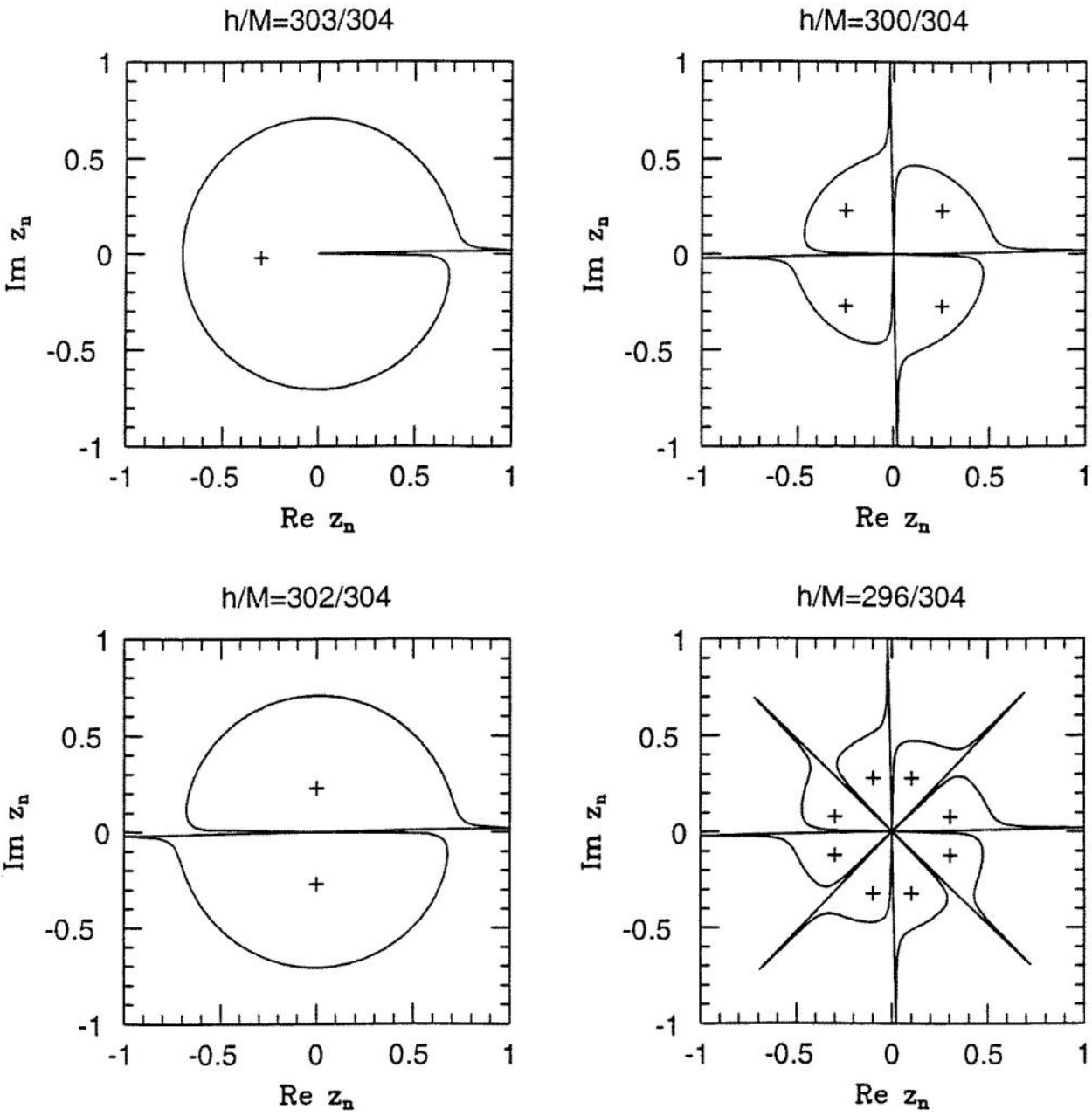

Figure 2. The closed trajectories of the spectral eigenvalues $\mathrm{f}(\mathrm{n})$ in the phasor scheme of WACR parametrized bySince, WACR are dense in theM $=\mathrm{k} 304$ and by different winding $\begin{array}{r}=303,302,300, \\ h /(M+1) \sim 1\end{array}$ numbers+ limit, we have chosen h,M such thath 296.

$$
\rightarrow-4
$$

The origin is at the vacuum state and sign of $\mathrm{f}(\mathrm{n})$ is always positive elsewhere.

The most important limiting case of WACR and ACR is $\mathrm{k}=-4^{+}$which implies $\mathrm{M} \rightarrow \infty$ such that $\mathrm{h} /(\mathrm{M}+1) \rightarrow 1^{-}$from equation (8). As the limit is sufficiently approached, the spectral eigenvalues [[n]] always acquire alternating signs and weakly admissible and admissible cyclic representations of the GHO are increasingly dense. At the limit, the spectral period of ACR as well as WACR tends to infinity and they are the only survivors in the spectrum. It will be shown in the next section that this limit is a particularly important resource for the understanding of problems with the harmonic-oscillator quantum phase at the infinite-period limit. We will now investigate a few physical applications of the (W)ACR in the formulation 
of an algebraic approach to quantum phase. In particular, the WACR developed here will be examined from this perspective in the section devoted to the index theorem.

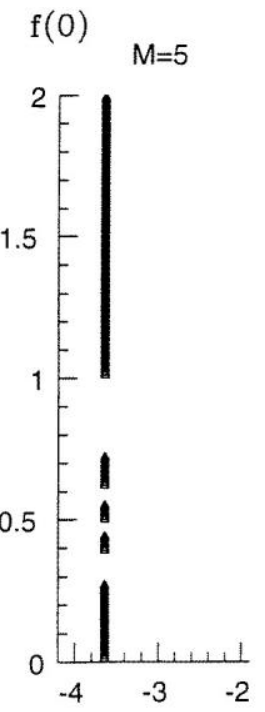

(a)

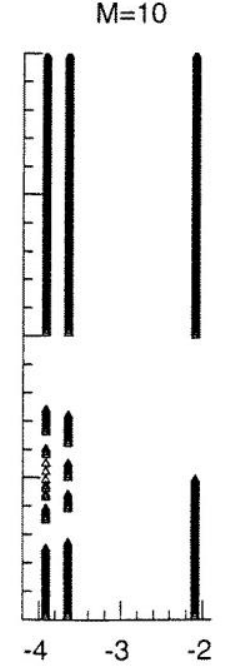

(b)

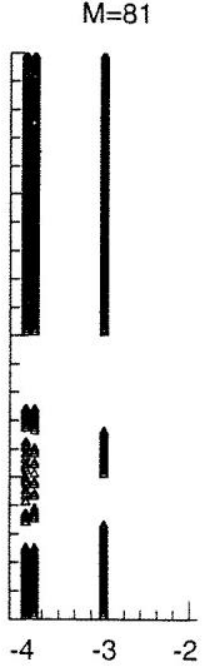

(c)

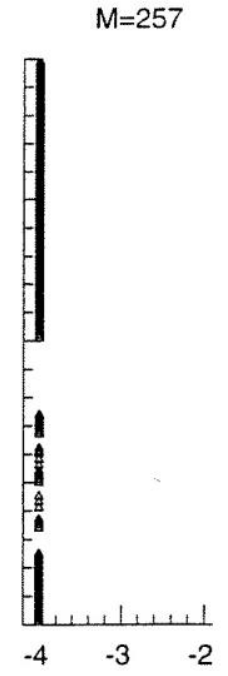

(d)

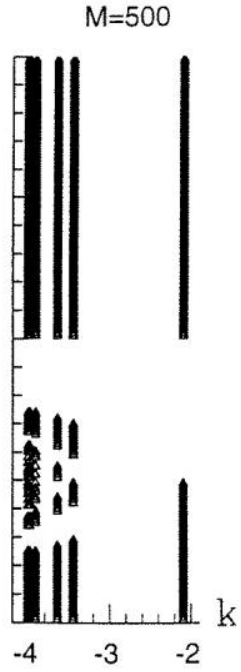

(e)

Figure 3. Bands of weakly admissible (i.e. $\mathrm{f}(0)=0$ and admissible (i.e. $0<\mathrm{f}(0)$ ) representations for different values of $f(0)$ and $k$ parametrized by $M=5,10,81,257,500$. The value of $h$ is varied with $\mathrm{k}$ according to equation (8).

\section{An algebraic approach to the quantum-phase problem}

In the formulation of the algebraic approach to quantum phase we will first follow a more generalized approach than the GHO and consider the most general admissible cyclic algebra as given by equations (1). The application of GHO to this problem will be investigated in section 3.1 where we will need a specific example. Let's consider the hth sector of the finitedimensional Fock space $\mathrm{F}^{(}{ }^{\mathrm{h}}{ }^{\mathrm{h}},(06 \mathrm{~h} 6 \mathrm{M})$ spanned by the vectors $\mid$ ni where $06 \mathrm{n} 6 \mathrm{M}$. The elements $a^{\wedge} a^{\wedge}+$ act on each finite-dimensional sector independently; hence, $a^{\wedge(h)}, a^{\wedge}{ }^{(h)}$. The polar decomposition of the generators in the hth sector is given by,

$$
\hat{a}^{(h)}=\hat{\mathcal{E}}_{\phi_{M}}^{(h)} \sqrt{\mathcal{F}(\hat{N})} \quad \hat{a}^{\dagger(h)}=\sqrt{\mathcal{F}(\hat{N})} \hat{\mathcal{E}}_{\phi_{M}}^{\dagger}
$$

where $^{\mathcal{F}(\hat{N})}=\mathcal{F}(\hat{N}+M+1)$ and we assume that the conditions for the WACR, namely, $06|\mathrm{~F}(\mathrm{~N})|$, or the ACR, namely, $0<\left|\mathrm{F}(\mathrm{N})^{\wedge}\right|$ are satisfied. The unitary phase operator acting on $\mathrm{F}^{(\mathrm{M})}$ is defined by,

$$
\hat{\mathcal{E}}_{\phi_{M}}^{(h)}=\sum_{m=1}^{M}|m-1\rangle\left\langle m\left|+\beta_{h}\right| M\right\rangle\langle 0|
$$

where $\left|\beta_{\mathrm{h}}\right|=1$, as in equation (1), is required for the unitarity of $\hat{\mathcal{E}}_{\phi_{M}}^{(\mathrm{h})}$, namely, $\hat{\mathcal{E}}_{\phi_{M}}^{(h)} \hat{\mathcal{E}}_{\phi_{M}}^{\dagger(h)}=1$ 
and $\left[\mathcal{E}_{\phi_{M}}^{(h)}, \mathcal{E}_{\phi_{M}}^{\dagger(h)}\right]=$

0 . With the conditions on the WACR or the ACR satisfied, the action

of equations (13) and (14) on the vectors in $\mathrm{F}^{(}{ }^{\mathrm{h}}$ ) complies with equations (1). The phase eigenstates,

$\hat{\mathcal{E}}_{\phi_{M}}^{(h)}|\phi\rangle_{h}=\mathrm{e}^{\mathrm{i} \phi_{h}}|\phi\rangle_{h} \quad|\phi\rangle_{h}=\frac{1}{\sqrt{M+1}} \sum_{m=0}^{M} \mathrm{e}^{\mathrm{i} m \phi_{h}}|m\rangle$

$\mathrm{h} / \mathrm{M}=80 / 81$

$06 \mathrm{~h} 6 \mathrm{M}$

$\mathrm{h} / \mathrm{M}=72 / 81$

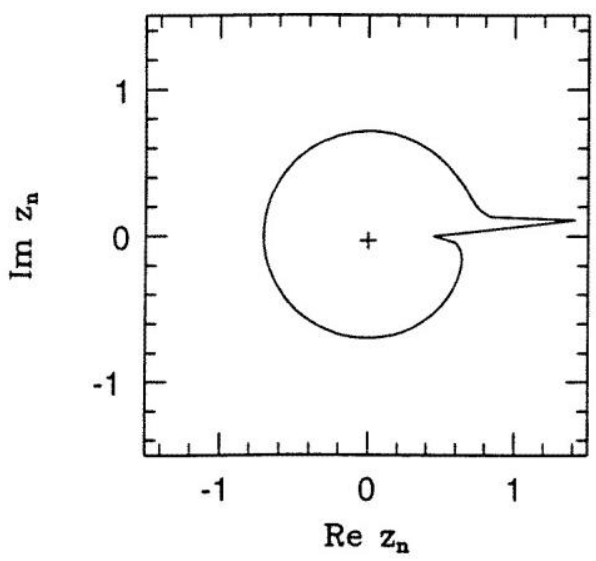

$\mathrm{h} / \mathrm{M}=78 / 81$

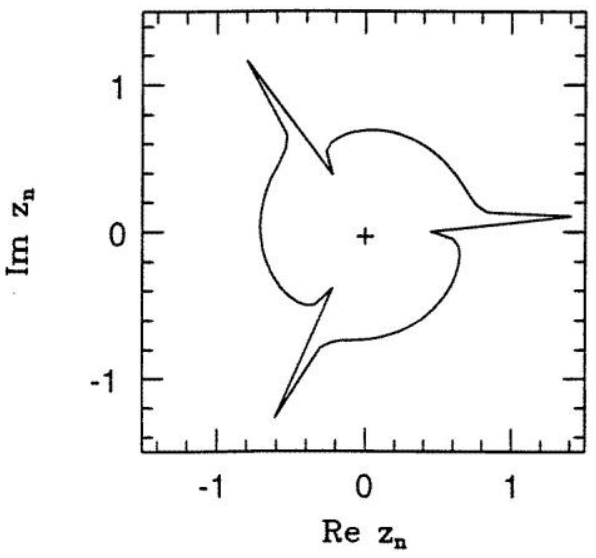

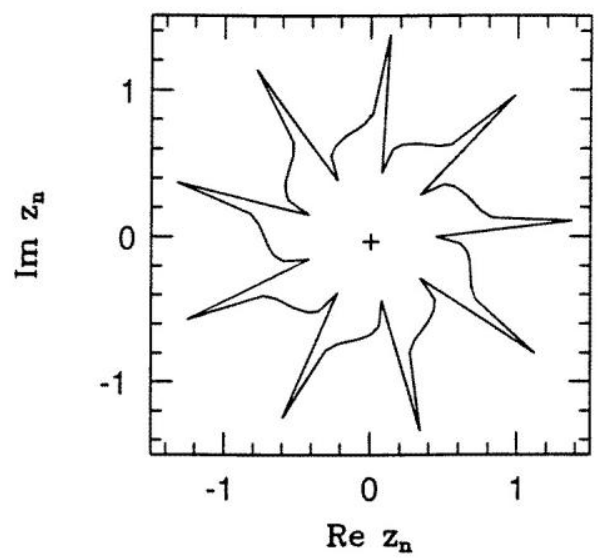

$\mathrm{h} / \mathrm{M}=54 / 81$

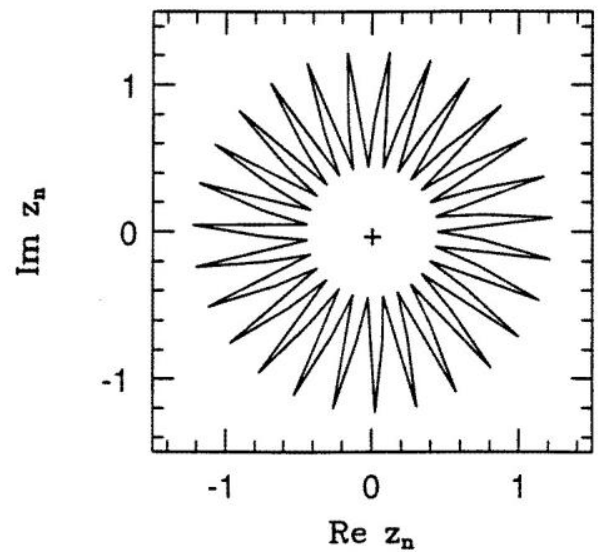

Figure 4. The closed trajectories of the spectral eigenvalues $f(n)$ in the phasor scheme of ACR parametrized by $M=81$ and $h=80,78,72,54$. The ACR are dense in the $k \rightarrow-{ }^{4}+$ limit, thus, in order to be able to sample more of them we have chosen $h, M$ such that $h /(M+1) \sim 1$. Other than conforming to $\mathrm{h} /(\mathrm{M}+1) \sim 1$, the values of $\mathrm{h}, \mathrm{M}$ can be arbitrary as in other figures which provides the freedom necessary to obtain an infinite variety of periods. Since $0<\mathrm{f}(0)$, there is no vacuum state in the spectra.

form an orthonormal complete set and resolve the identity as, 


$$
\text { ho h } \varphi \mid \varphi \text { ih }=\delta \text { h,ho } \quad \text { and } \quad I=X \mid \varphi \text { ih hh } \varphi \mid
$$

with $\varphi_{\mathrm{h}}=2 \pi \mathrm{h} /(\mathrm{M}+1)+\theta_{0}$ and $\beta_{\mathrm{h}}=\mathrm{e}^{\mathrm{i}(\mathrm{M}+1) \varphi_{\mathrm{h}}}$ where $\theta_{0}$ is an arbitrary reference phase. Because of the fact that the underlying representation is admissible (in accord with equation (1)) equations (13)-(16), define an algebraic approach to the quantum-phase problem. The orthonormality and the quantization of phase eigenstates and eigenvalues are natural algebraic consequences of the WACR or the ACR.

The equations (13)-(16) are in the same form as suggested by Pegg and Barnett [7]. The reason is that the form of the unitary phase operator in equation (14) can be written independently from the condition of admissibility of the underlying algebra [8]. The admissibility is required for the polar decomposition in equation (13). In particular, the Pegg-Barnett phase operator has the form

$$
\hat{\Phi}_{M}=\sum_{h=0}^{M} \phi_{h}|\phi\rangle_{h}{ }_{h}\langle\phi|=\frac{2 \pi}{M+1}\left\{\frac{M}{2} \mathbb{I}+\sum_{\substack{m, n=0 \\ m \neq n}}^{M} \frac{\mathrm{e}^{\mathrm{i}(m-n) \theta_{0}}|m\rangle\langle n|}{\mathrm{e}^{\mathrm{i}(n-m) 2 \pi /(M+1)}-1}\right\}
$$

and the phase-generalized number commutator is,

$$
\left[\hat{\Phi}_{M}, \mathcal{F}(\hat{N})\right]=\frac{2 \pi}{M+1} \sum_{\substack{m, n=0 \\ m \neq n}}^{M} \mathrm{e}^{\mathrm{i}(m-n) \theta_{0}} \frac{[f(n)-f(m)]|m\rangle\langle n|}{\mathrm{e}^{\mathrm{i}(n-m) 2 \pi /(M+1)}-1} .
$$

The Hermitian phase operator in equation (17) and the unitary cyclic one in equation (15) have diagonal representations in the same phase eigenspace. In the algebraic approach presented here, the Hermitian Pegg-Barnett phase operator is replaced by the unitary one in (15). As a result, the commutator in equation (18) is also replaced accordingly by

$\left[\hat{\mathcal{E}}_{\phi_{M}}^{(h)}, \mathcal{F}(\hat{N})\right]=\sum_{m=h+1}^{h+M}[f(m)-f(m-1)]|m-1\rangle\left\langle m\left|+\beta_{h}[f(0)-f(M)]\right| M\right\rangle\langle 0|$.

In contrast to the Pegg-Barnett approach, where the cyclic property of the phase operator is recovered at the commutation relation in equation (18), in the algebraic approach, the admissible cyclic property of the representation is retained from the beginning at the operator level. In fact, it can be seen from equations (14) and (15) that, the identities $\hat{\mathcal{E}}_{\phi_{M}}^{(h)} \equiv \hat{\mathcal{E}}_{\phi_{M}}^{\left(h+h_{0}\right)}$ where $h_{0}=M+1$ and $f(n) \equiv f(n+M+1)$ indicate this cyclic property.

The underlying cyclic representation and the fact that the identity operator is now cyclic (i.e. $\mathbb{I}=\sum_{n=0}^{M}|n\rangle\left\langle n\left|\equiv \sum_{n=n_{0}}^{M+n_{0}}\right| n\right\rangle\langle n|$ ) allow equation (14) to be written in a conventionally more appealing form for the case when $\theta_{0}=2 \pi \mathrm{r} /(\mathrm{M}+1)$, for $(06 \mathrm{r} 6 \mathrm{M})$. For this case we observe that $\beta_{\mathrm{h}}=1$ and,

$$
\hat{\mathcal{E}}_{\phi_{M}}^{(h)}=\sum_{m=h+1}^{h+M+1}|m-1\rangle\langle m|
$$

For these set of $\theta_{0}$ values, the commutation in equation (19) becomes

$$
\left[\hat{\mathcal{E}}_{\phi_{M}}^{(h)}, \mathcal{F}(\hat{N})\right]=\sum_{m=h+1}^{h+M+1}[f(m)-f(m-1)]|m-1\rangle\langle m| .
$$

If we apply the admissible cyclic GHO algebra in section II to equation (21), the standard harmonic oscillator result is recovered in the infinite period limit of NCR (i.e. $\mathrm{k}=0^{-}$) which is known as the Susskind-Glogower-Carruthers-Nieto phase-number commutation relation [9]. In this limit, equation (14) is also well defined and approaches to the unitary version of 
the Pegg-Barnett phase operator. However, the condition on admissibility is not respected for NCR in this limit. One of the important consequences is that, the standard harmonic oscillator is realized as the infinite-period limit of a NCR. We will examine the properties of the phase operator using the (W)ACR in the next section devoted to the index theorem.

The properties and action of the operator in equation (20) on the vectors in the finitedimensional Fock space is identical to those of (14). Equation (20) implies that in each sector $\mathrm{F}^{(} \mathrm{M}^{\mathrm{h})}$, there is a well-defined unitary phase operator and its eigenstate $\mid \varphi$ in is a superposition of all vectors in $\mathrm{F}^{(}{ }^{\mathrm{h}}$ ) as given by equation (15). This amounts to associating with each sector $\mathrm{F}_{\mathrm{M}}^{(\mathrm{h})}$ a phase eigenvalue $\varphi_{\mathrm{h}}$ and an eigenstate $\mid \varphi_{\mathrm{h}}$. The connection in the phase eigenspace between the orthogonal sectors $\mathbb{F}_{M}^{(h)}$ and $\mathbb{F}_{M}^{(h+1)}$ is provided by the discrete phase translation operator

$$
\mathrm{e} \pm \mathrm{i} 2 \pi \mathrm{N} /\left(\mathrm{M}^{\wedge} \quad+1\right)|\varphi \mathrm{ih}=| \varphi \mathrm{ih} \pm 1
$$

whereas a continuous translation by an arbitrary $\gamma_{0}$ produces phase superposition states,

$$
\mathrm{e}^{\mathrm{i} \gamma_{0} \hat{N}}|\phi\rangle_{h}=\sum_{h^{\prime}=0}^{M} \Gamma_{h h^{\prime}}\left(\gamma_{0}\right)|\phi\rangle_{h^{\prime}} \quad \Gamma_{h h^{\prime}}\left(\gamma_{0}\right)=\frac{1}{M+1} \sum_{m=0}^{M} \mathrm{e}^{\mathrm{i} m\left(\phi_{h}-\phi_{h^{\prime}}+\gamma_{0}\right)} .
$$

The $\gamma_{0}$ translated phase states in (23) are no longer eigenstates of the unitary phase operator in (14) or (20) unless $\gamma_{0}=2 \pi \mathrm{r} /(\mathrm{M}+1)$. The choice of $\gamma_{0}$ also provides a physical interpretation of the arbitrary reference phase $\theta_{0}$. A particular choice of $\theta_{0} 6=0$ relative to $\theta_{0}=0$ is equivalent to a particular reference of the absolute orientation of the orthogonal phase eigenstates. The continuous rotation of this absolute frame is generated by the unitary operation in equation (23). A particular choice of $\gamma_{0}$ determines the axis of rotation which is given by the orientation of the invariant unit vector |vi:

$$
\mathrm{e}^{\mathrm{i} \gamma_{0} \mathrm{~N}^{\wedge}}\left|\mathrm{vi}=\mathrm{e}^{\mathrm{i} \lambda}\right| \mathrm{vi} \quad \text { where } \quad|v\rangle=\sum_{h=0}^{M} v_{h}|\phi\rangle_{h} \quad \mathrm{k} \mid \mathrm{vik}=1 .
$$

For a particular choice of $\gamma_{0}$, the direction of rotation is specified by the invertible condition

$$
v_{h}=\mathrm{e}^{-\mathrm{i} \lambda} \sum_{h^{\prime}=0}^{M} v_{h^{\prime}} \Gamma_{h^{\prime} h}\left(\gamma_{0}\right) \quad 06 \mathrm{~h} 6 \mathrm{M} .
$$

Equation (25) is an eigenvalue equation for $\lambda$ and defines a linear set of $(M+1)$ equations which can be solved for a given $\gamma_{0}$. From equations (24) and (15) it is also clear that |vi cannot be an eigenstate of the phase operator in (14) or (20). In fact it is obvious from equation (24) that |vi is a pure number state. Thus, |vi fluctuates around its expected orientation as observed from the phase eigenspace. The projection of its components on the phase eigenspace yield broadened probability distributions given by

$$
\left.\left.\right|_{h}\left\langle\phi\left|\mathrm{e}^{\mathrm{i} \gamma_{0} \hat{N}}\right| \phi\right\rangle_{h^{\prime}}\right|^{2}=\frac{1}{(M+1)^{2}} \frac{1-\cos (M+1)\left(\phi_{h}-\phi_{h^{\prime}}+\gamma_{0}\right)}{1 \underbrace{\cos \left(\phi_{h} \phi_{h^{\prime}}+\gamma_{0}\right)}_{-}}
$$


The equation (26) yields a nonfluctuating distribution only when $\gamma_{0}=2 \pi \mathrm{r} /(\mathrm{M}+1)$ leading $\operatorname{to}^{\mathrm{M}}|\mathrm{hh} \varphi| \mathrm{e}^{\mathrm{i} \gamma_{0} \mathrm{~N}^{\wedge}}|\varphi \mathrm{iho}|^{2}=\delta_{\mathrm{h} 0, \mathrm{~h}+\mathrm{r} .}$ On the other hand, for an arbitrary normalized state $\left|9^{\mathrm{i}}{ }^{\mathrm{P}} \mathrm{P}_{\mathrm{r}=0} \psi_{\mathrm{r}}\right| \mathrm{ri}$, where $\mid \mathrm{ri}_{\left\{0_{6} \mathrm{r}_{6} \mathrm{M}\right\}}$ are vectors in $\mathrm{FM}$, the phase probability distribution as observed in a general phase reference frame identified by $\gamma_{0}$ can be written as

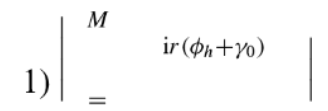

$$
\begin{aligned}
& P\left(\phi_{h}+\gamma_{0}\right)=\left.\left.\right|_{h}\left\langle\phi\left|\mathrm{e}^{\mathrm{i} \gamma_{0} \hat{N}}\right| \Psi\right\rangle\right|^{2}=\frac{1^{\theta_{0}}}{(M+} \sum_{r 0} \mathrm{e}^{-} \quad \psi_{r}{ }^{2} \quad 06 \mathrm{~h} 6 \mathrm{M}
\end{aligned}
$$

where $\gamma_{0}$ redefines the reference phase. A comparison of equations (26) and (27) yields that $\gamma_{0}$ should be interpreted on the same footing as $\theta_{0}$ which then verifies that the phase fluctuations in an arbitrary state $\mid 9 \mathrm{i}$ are strongly susceptible to the choice of $\theta_{0}$. In order to show this effect more specifically, we consider an overcomplete expansion of the state $9 \mathrm{i}$ over a continuous distribution $\mathrm{P}\left(\gamma_{0}\right)$ as,

$$
\mid 9 \mathrm{i}=\mathrm{Z} \text { d } \gamma_{0} \mathrm{P}\left(\gamma_{0}\right) \mathrm{e}_{\mathrm{i} \gamma 0 \mathrm{~N}^{\wedge}} \mid \varphi \mathrm{iho} \quad \mathrm{k} 9 \mathrm{ik}=1
$$

based on a fixed $\mid \varphi$ iho. In this case the phase probability distribution $\mathrm{P}\left(\varphi_{\mathrm{h}}\right)$ is given by

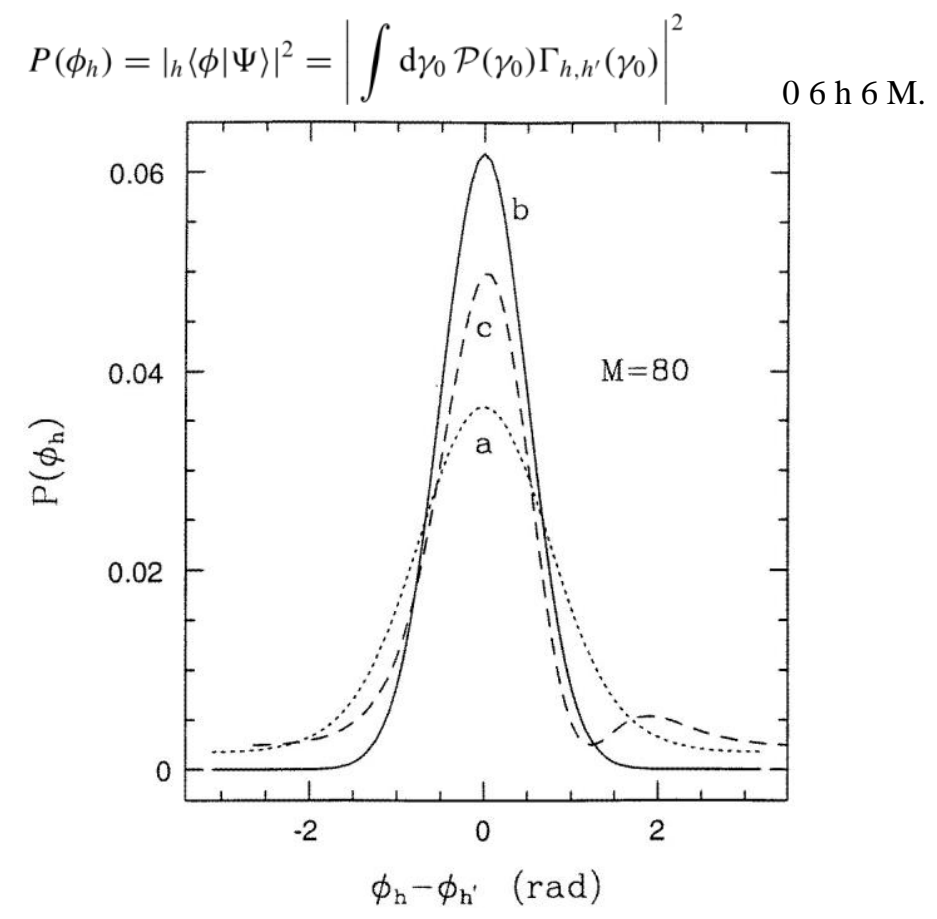

Figure 5. The effect of the arbitrariness of the reference phase on the phase distribution $\mathrm{P}\left(\varphi_{\mathrm{h}}\right)$ for $M=80$. Here (a) represents the maximal effect when $\gamma_{0}$ is distributed continuously over the range 
$-\pi 6 \gamma_{0} 6 \pi$, (b) corresponds to the case when $\gamma_{0}=2 \pi /(\mathrm{M}+1)$ with the complete set $06 \mathrm{r} 6 \mathrm{M}$ and

(c) represents the case when $\gamma_{0}$ varies over the complete set as in (b) but shifted by an arbitrary phase $\alpha$. Here $\alpha=\pi / 3$.

Equation (29) implies that the phase probability in $\mid 9 \mathrm{i}$ is not only influenced by the continuous distribution $\mathrm{P}\left(\gamma_{0}\right)$ but is also broadened by the factor $0_{\mathrm{hh}}\left(\gamma_{0}\right)$. This superfluous broadening factor is obviously an artefact of the wrong choice of the basic phase coordinates in (28). The broadening factor remains even if equation (28) is represented in a complete basis with $\gamma_{0}=$ $2 \pi \mathrm{r} /(\mathrm{M}+1)+\alpha$ with $06 \mathrm{r} 6 \mathrm{M}$ where $\alpha \in \mathrm{R}$ otherwise arbitrary.

The calculations for $\mathrm{P}\left(\varphi_{\mathrm{h}}\right)$ are shown in figure 5 for three independently normalized $\mathrm{P}\left(\gamma_{0}\right)$ distributions when: $\left(\mathcal{P}\left(\gamma_{0}\right)=\mathcal{N}_{1} \exp \left(-\gamma_{0}^{2}\right)\right.$ a) is a continuous distribution over the range $-\pi$ $6 \gamma_{0} 6 \pi$ represented by the dotted curve; (b) the discrete distribution

$\mathcal{P}\left(\gamma_{0}\right)=\mathcal{N}_{2} \exp \left(-\gamma_{0}^{2}\right) \sum_{r=0}^{M} \delta\left[\gamma_{0}-2 \pi r /(M+1)\right]$ represented by the full curve and, (c) the discrete distribution $\mathcal{P}\left(\gamma_{0}\right)=\mathcal{N}_{3} \exp \left(-\gamma_{0}^{2}\right) \sum_{r=0}^{M} \delta\left[\gamma_{0}-2 \pi r /(M+1)-\alpha\right]$, where $\alpha$ is not a multiple of $2 \pi /(M+1)$, is represented by the broken curve. The peak position of the phase distribution in (c) is shifted and asymmetric because of the $\alpha 6=0$ where we considered in figure 5 the case $\alpha=\pi / 3$. The $\mathrm{Ni}^{\text {'s }}$ for $\mathrm{i}=1,2,3$ are appropriate normalizations so that

$\int_{-\pi}^{\pi} \mathrm{d} \gamma_{0} \mathcal{P}^{2}\left(\gamma_{0}\right)=\quad 1$. In order to compare the widths of all cases (a), (b) and (c) the peak position of the third distribution is shifted to zero. The results confirm that the choice of the reference phase is generally influencial on the phase distribution. In particular, when $\gamma_{0}$ is a multiple of the phase quantum (i.e. case (b)) the broadening effect is minimized. In this case, and putting $\gamma_{0}$ and $\theta_{0}$ on the same footing, we notice that the phase operator adopts its more conventional form in equation (20). The $\mathrm{M} \rightarrow \infty$ limit in equation (29) deserves a

distribution is purely determined by that of the stateparticular attention. In this limit we have $\operatorname{limm} \rightarrow \infty_{0} 0$ h $\mid 9_{0}(\gamma$ io $)$ i.e. $=\mathrm{P}(\varphi) \delta\left(\varphi^{-}=\varphi^{0} \mathrm{P}+2 \gamma\left(\varphi^{0}\right)_{0}-\right.$ and the phase $\left.\varphi\right)$. Hence,

at the infinite period limit the phase probability distribution is immune to the choice of the reference phase.

Before we discuss the application of this algebraic approach in the next section it should be stressed that one can also generalize equation (22) and (23) and their consequences to rotations in the finite-dimensional number operator eigenspace using the unitary operator $\left(\hat{\varepsilon}_{\phi_{M}}^{+}\right)^{n_{0}}$ where $n_{0} \in Z$. Let us denote the complete set of orthonormal phase vectors $\{\mid \varphi \mathrm{ih}\}_{06 \mathrm{~h}_{6} \mathrm{M}}$ as elements of the $(\mathrm{M}+1)$-dimensional phase space Рм. Then Рм provides the basis vectors of the dual representation to the finite-dimensional Fock space FM studied previously in this section. Similar to the ( ${ }^{(\mathrm{h})} \mathrm{M}+1$ )-independent sectors labelled by (h) in $\mathrm{F}_{M}$, the dual space PM has $(\mathrm{M}+1)$ independent sectors where we denote each independent sector by $\mathrm{P}^{(} \mathrm{M}^{\mathrm{n})}$ with $06 \mathrm{n}$ $6 \mathrm{M}$. For each independent sector n, a Fock vector |ni can be associated such that

$$
|n\rangle=\frac{1}{\sqrt{M+1}} \sum_{h=n}^{n+M} \mathrm{e}^{-\mathrm{i} n \phi_{h}}|\phi\rangle_{h} \quad\{\mid \varphi \mathrm{ih}\}_{\mathrm{n} 6 \mathrm{~h} 6 \mathrm{n}+\mathrm{M}} \in \mathrm{P}^{(} \mathrm{M}^{\mathrm{n})} .
$$


Similar to the equation (22), here, the action of the operator $\left(\hat{\varepsilon}_{\phi_{M}}^{+}\right)^{n_{0}}$ is to connect two different sectors of the dual space $\mathbb{P}_{M}^{(n)}$ and $\mathbb{P}_{M}^{\left(n+n_{0}\right)}$ as

$$
\left(\hat{\varepsilon}_{\phi_{M}}^{+}\right)^{n_{0}}|n\rangle=\left|n+n_{0}\right\rangle \text {. }
$$

For arbitrary $\mathrm{n}_{0}$ one obtains a finite set of generalized representations where $\mathrm{n}_{0}$ denotes the index of the reference state. For $\mathrm{n}_{0}=0$ the conventional Fock-space representation is recovered. The further implications of this dual picture between the representations in Рм and FM are outside the scope of this work and we will not pursue this formalism here.

Coming back to the initial problem after this short excursion into the dual picture, we will show in the next section that there exists a singular infinite-period limit in the spectrum of (W)ACR which identifies the incurable anomaly of the harmonic-oscillator quantumphase operator. In order to examine this limit, we use a prototype nonlinear oscillator which is derived from the GHO, of which the spectrum is of WACR type, yielding the standard harmonic oscillator at the very limit $\mathrm{M}=\infty$. This particular oscillator will then be used as a tool to demonstrate in its spectrum, a singular behaviour of the limit at $\mathrm{M}=\infty$. We discuss the consequences of this result and verify that the harmonic oscillator does not provide the appropritate basis to search for the Hermitian quantum phase algebraically. This, however, is not a proof based on a uniqueness theorem; neither do we suggest that, the GHO is the most general oscillator where WACR or ACR can be found. Whether there are other admissibly cyclic algebras which yield the standard harmonic oscillator in their proper limit, is a legitimate question from the index-theorem point of view which was recently examined by Fujikawa [10] for the free-photon field. We will now investigate the implications of the index theorem on equations (13)-(16) within the context of (W)ACR.

\subsection{The index theorem}

Fujikawa examined the index condition for the free photon fields $b,^{\wedge} b^{\wedge}$ by

$$
\mathrm{I}=\operatorname{Tr}\left\{\mathrm{e}-\mathrm{b}^{\wedge}+\mathrm{b} / \mathrm{c}^{\wedge} 2\right\}-\operatorname{Tr}\left\{\mathrm{e}-\mathrm{b}^{\wedge} \mathrm{b}^{\wedge}+/ \mathrm{c}_{2}\right\}
$$

where $\mathrm{c}$ is a real but arbitrary constant. The equation (32) puts a stringent condition on I for the unitarity of the photon phase operator $\mathrm{E}(\varphi)^{\wedge}$. If the free photon fields were polar decomposed into

$$
\mathrm{b}^{\wedge}=\mathrm{E}(8)^{\wedge} \mathrm{p}^{\wedge} \mathrm{N}^{\wedge} \quad \mathrm{b}^{\wedge} \uparrow==\mathrm{p}^{\wedge} \mathrm{N}^{\wedge} \mathrm{E}^{\wedge}(8)^{\wedge} \quad=
$$

with $\mathrm{N}^{\wedge}$ as Hermitian and $\mathrm{E}(8) \mathrm{E}^{\dagger}(8) \mathrm{E}^{\dagger}(8) \mathrm{E}(8) 1$ as required by the unitarity, the trace identity ensures that $\mathrm{I}=0$ independently from $\mathrm{c}$. Here, we made a formal distinction between $\mathrm{E}(8)^{\wedge}$

for the free-photon field and $\hat{\mathcal{E}}_{\phi}{ }_{\wedge}$ in equation (14) and (20). On the other hand, the direct application of the commutator $\left[\mathrm{b}, \mathrm{b}^{\dagger}\right]=1$ with the vacuum condition $\mathrm{b}^{\wedge} \mid 0 \mathrm{i}=0$ implies that $\mathrm{I}=$ 1 restating Dirac's original paradox in a different language. The paradox clearly shows the impossibility of recovering a unitary phase operator in Dirac's approach. The index I simply counts the difference in the number of zero eigenvalues in the spectrum of $b^{\wedge} b^{\wedge}$ and $b^{\wedge} b^{\wedge}+$ and 
it is clearly robust to any unitary transformation on $b,^{\wedge} b^{\wedge}$. A quick remedy can be provided by folding the infinite-dimensional Fock space into an infinitely many independent finite sectors (i.e. h) of M dimensions and connecting the ends of each piece so that,

$$
\begin{aligned}
& \hat{E}(\Phi)=\sum_{n=1}^{M}|n-1\rangle\left\langle n\left|+\beta_{h}\right| M\right\rangle\langle 0| \\
& \hat{b}=\hat{E}(\Phi) \sqrt{\hat{N}}=\sum_{n=1}^{M} \sqrt{n}|n-1\rangle\langle n|
\end{aligned}
$$

and similarly for $\mathrm{b}^{\wedge}$. The index is as required (i.e. $\mathrm{I}=0$ ), the phase is unitary and the polar decomposition is satisfied. Equation (34) is basically the starting point of the Pegg-Barnett approach to truncated Fock spaces. A comparison between the phase operator in (34) and that obtained from (W)ACR in equation (14) shows that they are very similar. Although the quantization of the phase eigenvalues in orthogonal phase eigenspaces is assumed in the Pegg-Barnett approach, they naturally arise as a result of cyclic property of the algebra as demonstrated in equations (13)-(16).

We now look for other examples of this algebraic approach for which the generally deformed cyclic algebra in equation (1) is a candidate. In fact, the condition for the vanishing index for a generalized oscillator in a finite-dimensional Fock space coincides with the condition for the periodic spectrum

$$
I=\operatorname{Tr}\left\{\mathrm{e}^{\mathcal{F}(\hat{N}) / c^{2}}\right\}-\operatorname{Tr}\left\{\mathrm{e}^{\mathcal{F}(\hat{N}+1) / c^{2}}\right\}=\sum_{n=0}^{M}\left\{\mathrm{e}^{f(n) / c^{2}}-\mathrm{e}^{f(n+1) / c^{2}}\right\}=0 \quad 06 \mathrm{f}(\mathrm{n}) \forall \mathrm{n}
$$

iff $f(0)=f(M+1)$. Here $F(N)^{\wedge}$ is the generalized number operator with eigenvalues $f(n)$ as defined below equation (1). Now using the expressions (13) and (14) the generators of any admissibly cyclic algebra can be constructed as,

$$
\hat{a}=\sum_{n=0}^{M} f^{1 / 2}(n)|n-1\rangle\left\langle n\left|+\beta_{h} f^{1 / 2}(M+1)\right| M\right\rangle\langle 0| .
$$

Equation (36) implies that the periodic vacuum condition is not in contradiction with the rest of the picture: $h 0\left|{ }^{\wedge} a^{\dagger} a^{\wedge}\right| 0 i=h M\left|{ }^{\wedge} a^{\wedge}\right| M i=f(0)=f(M+1)$. Hence the $a^{\wedge}{ }^{\wedge} a^{\wedge}$ and $a^{\wedge} a^{\wedge}{ }^{\dagger}$ operators have identical spectra which make the index vanish. For instance, the specific ACR of the qdeformed oscillator has been examined in [11] in this context.

Since the condition on the periodic spectrum for the existence of the unitary phase is strict, one has to be careful in calculating the index in a particular algebra. The period $\mathrm{M}$ can be arbitrarily large but not infinite before the end of all calculations since the $\mathrm{M} \rightarrow \infty$ limit is singular at $\mathrm{M}=\infty$. Infact, this recipe is also independent of the algebra. For instance, from equation (11), at the $\mathrm{M} \rightarrow \infty$ limit the WACR with $\mathrm{f}(0)=0$ yields the spectrum $\mathrm{f}_{\infty}(\mathrm{n})=\mathrm{n} /(2 \mathrm{n}$ -1). A direct application of this to calculate the index yields $I=1$; whereas, for finite $\mathrm{M}$ one obtains a result $\mathrm{I}=0$ independent of $\mathrm{M}$.

Let us consider the composition $f \circ f(n)$ using the spectrum in equation (11) taking it as a simple mathematical tool in the analysis of the infinite-period limit. We first construct $f_{\infty} \circ$ $\mathrm{f}_{\infty}(\mathrm{n})$ as, 


$$
f_{\infty} \circ f_{\infty}(n)=\frac{\frac{n}{(2 n-1)}}{2 \frac{n}{(2 n-1)}-1} \equiv n .
$$

Hence, one obtains the harmonic oscillator spectrum. The singularity at the infinite period limit becomes transparent if the order of the limiting process is changed. If $\mathrm{M} \rightarrow \infty$ limit is taken at the last stage by using equation (11), one finds that

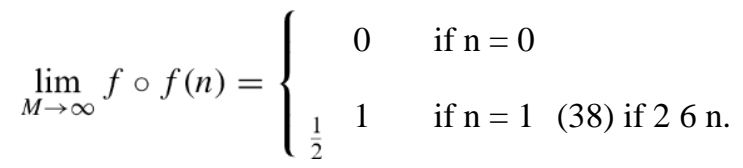

For finite $M$ it is clear from equation (11) that $f \circ f(n)=f \circ f(n+M+1)$. The spectrum of $f \circ$ $\mathrm{f}(\mathrm{n})$ is always positive and cyclic provided that $\mathrm{f}(\mathrm{n})$ is positive and cyclic for all $\mathrm{n}$. This implies that, the spectrum $f \circ f(n)$ corresponds to yet another generally deformed oscillator with a WACR of which singular limit at $\mathrm{M}=\infty$ yields the standard harmonic oscillator. Whether there are more examples of such nonlinear oscillators with this property at $M=\infty$ is currently an open question for the author. Although the oscillator $f \circ f(n)$ is not of much practical importance, it gives a clear demonstration of the connection between the two problems, namely the absence of a Hermitian quantum phase and the singular behaviour of the spectrum at the infinite-period harmonic-oscillator limit. Hence, the standard harmonic oscillator's linear spectrum is placed on the singular infinite period limit of the NCR in the case of GHO or of the ACR in the case of the algebra of which spectrum is the composition given by equation (37). This result naturally renders any truncation and limiting scheme within an infinite-dimensional Fock space (although it might be convenient from the practical calculation point of view) as nonalgebraic and unnatural.

\section{Conclusions}

In this work we have investigated various cyclic representations of the GHO and used it as a tool to analyse the properties of the quantum-phase operator in an algebraic perspective. We have shown that, in certain ranges of the deformation parameter, the generalized homographic oscillator produces a rich variety of cyclic representations among which the weakly admissible and the admissible ones are particularly important tools from the phase operator point of view. The algebraic properties of the phase operator in generalized ACR are studied. The dependence of the phase fluctuations on the choice of reference frame in the phase eigenspace is confirmed and shown that, if the reference phase is also quantized in units of the fundamental quantum phase the superfluous phase fluctuations arising from the arbitrariness of reference phase can be minimized. For this choice of reference phase, the phase operator also takes its Susskind-Glogower-Carruthers-Nieto-type conventional form.

The problems in constructing a Hermitian quantum phase in the harmonic oscillator algebra is reaffirmed to be connected with the nonalgebraic singular behaviour at infiniteperiod limit. This result is tested on a new spectrum (i.e. equations (37) and (38)) which is admissibly cyclic with the singular limit properly coinciding with the harmonic oscillator. The combination of several restrictions such as admissibility and the existence of a polar decomposition of the elements require that one has to stay within an admissibly cyclic representation and let the size of the corresponding Hilbert space approach to infinity at the final level of calculations. In this respect, we suggest as a side remark that, the conceptual 
basis underlying the limiting procedure which was first examined by Lerner et al [12] and explicitly considered by Pegg and Barnett [7] in connection with the phase problem in quantum optics can also be made transparent, from this formal algebraic point of view, as a recipe to avoid the singular ambiguity in the infinite spectrum before the calculations are finalized.

There are a number of other interesting properties of the ACR. In particular, the construction of their minimum-uncertainty states and the properties of quantum fluctuations in these states are currently being examined. From a statistical point of view, they also provide a generalized outlook onto the concept of parastatistics a la Green [13] as well as' Greenberg and Mohapatra [14]. These are out of the current scope of this work which might be addressed in another publication.

\section{Acknowledgments}

The author thanks especially Professor M Arık, with whom some of the preliminaries of the GHO were developed previously [5] and Professor T Dereli and Professor A Verc, in for useful discussions. He also thanks the referee for pointing out [15] where a generalized treatment of admissible algebras is presented. In the context of this reference, the (W)ACR examined here fall into the class of nondegenerate finite-dimensional cyclic representations of the generalized 'ucr' algebra therein.

\section{References}

[1] De Concini C and Kac V G 1991 Prog. Math. 92471

[2] Dobrev V K 1991 Symmetries in Science V: Algebraic Systems, their Representations, Realizations, and Physical Applications ed B Gruber et al (New York: Plenum)

[3] Abdesselam B, Arnaudon D and Chakrabarti A 1995 J. Phys. A: Math. Gen. 285495

[4] Hong-Chen Fu and Ryu Sasaki 1996 J. Phys. A: Math. Gen. 294049

[5] Hakioglu T and Arık M 1996 Phys. Rev. A 5452

Hakioglu T and Arık M 1997 Generalized homographic oscillator in the stereographic deformation of SU(2) and SU(1,1) Phys. Scr. submitted

[6] Ellinas D 1992 Phys. Rev. A 453358

[7] Pegg D T and Barnett S M 1988 Europhys. Lett. 6483

Pegg D T and Barnett S M 1989 Phys. Rev. A 391665

[8] Halmos P R 1982 A Hilbert Space Problem Book (Berlin: Springer)

[9] Susskind L and Glogower J 1964 Physics 149

Carruthers P and Nieto M M 1965 Phys. Rev. Lett. 14387

Carruthers P and Nieto M M 1965 Rev. Mod. Phys. 40411

[10] Kazuo Fujikawa 1995 Phys. Rev. A 523299

[11] Kazuo Kujikawa, Kwek L C and Oh C H 1995 Mod. Phys. Lett. A 102543

[12] Lerner E C, Huang H W and Walters G E 1970 J. Math. Phys. 111679

[13] Green H S 1953 Phys. Rev. 90270

[14] Greenberg O W and Mohapatra R N 1987 Phys. Rev. Lett. 592507

[15] Dubin D A, Hennings M A and Solomon A I 1997 J. Math. Phys. 383238 